\title{
LOCAL VARIETIES AND ASYMPTOTIC EQUIVALENCE
}

\section{H. T. MUHLY}

1. Let $\mathfrak{o}$ be a local domain and let $\mathfrak{a}$ be an ideal of $\mathfrak{o}$ that is primary for the maximal ideal $\mathfrak{m}$ of $\boldsymbol{o}$. If $\alpha$ is an element that is superficial of degree $s$ for $\mathfrak{a}$ (in the sense of $\left[6\right.$, p. 22]) and $\alpha_{1}, \cdots, \alpha_{t}$ is a basis for $\mathfrak{a}^{s}$, let $\mathfrak{o}(\mathfrak{a}, \alpha)=\mathfrak{o}\left[\alpha_{1} / \alpha, \cdots, \alpha_{t} / \alpha\right]$. If $\mathfrak{m}^{\prime}$ is a prime ideal of $\mathfrak{o}(\mathfrak{a}, \alpha)$ such that $\mathfrak{m}^{\prime} \cap \mathfrak{o}=\mathfrak{m}$, then the quotient ring of $o(a, \alpha)$ at $\mathfrak{m}^{\prime}$ is called an $\mathfrak{a}$-spot, and the totality of $\mathfrak{a}$-spots is called the variety $V(\mathfrak{a})$ of $\mathfrak{a}$. Let $\Omega$ be the set of all valuations of the quotient field $F$ of $\mathfrak{o}$ that dominate $\boldsymbol{o}$. It is shown that $V(\mathfrak{a})$ is a complete variety relative to $\Omega$ in the sense that each element $v$ of $\Omega$ dominates a unique $a$-spot. If $\mathfrak{D}$ is analytically normal it is shown that for each $m$-primary ideal $\mathfrak{a}$ there is an integer $g$ such that the integral closure $\left(\mathfrak{a}^{0}\right)_{a}$ of $\mathfrak{a}^{0}$ is such that all of its powers are integrally closed. Ideals with this property are called normal and $\left(a^{0}\right)_{a}$ is called a derived normal ideal of $\mathfrak{a}$ and denoted by $\mathfrak{a}_{g}$. If an ideal is normal, then its variety is normal and $V\left(\mathfrak{a}_{\mathfrak{a}}\right)$ is a normalization of $V(\mathfrak{a})$ in the sense that the integral closure in $F$ of any spot $P$ of $V(a)$ is the intersection of a finite number of spots in $V\left(\mathfrak{a}_{0}\right)$ that dominate $P$. In $\S 3$ it is shown that there is a $1: 1$ correspondence between the algebraic points of the variety of the null-form ideal of $\mathfrak{a}$ (defined over the residue field $k$ of $\mathfrak{o}$ ) and the set of $\mathfrak{a}$-spots of maximal rank. Hence it is not surprising that $V(\mathfrak{a})$ should have properties analogous to those of varieties over a field. In $\$ 4$ some of these properties are elaborated. In particular, the usual relation of dominance between varieties $V(\mathfrak{a})$ and $V(\mathfrak{b})$ yields a partial order on the set of $m$-primary ideals that directs the set. If $P$ and $P^{\prime}$ are a-spots such that $P$ is a quotient ring of $P^{\prime}$ then we say that $P^{\prime}$ is a specialization of $P$. For each $m$-primary ideal $a$ there is a finite set $\left\{P_{1}, P_{2}, \cdots, P_{s}\right\}$ of $\mathfrak{a}$-spots such that each $\mathfrak{a}$-spot is a specialization of one of the $P_{i}$. An irreducible ideal is one whose spots are all specializations of a single spot $P$ which is then called a general spot for the ideal. Finally, we show that the notion of asymptotic equivalence in the projective sense introduced by Samuel in [7] can be characterized in terms of local varieties. In fact, if $\mathfrak{a}$ and $\mathfrak{b}$ are asymptotically equivalent in the projective sense (in symbols: $\mathfrak{a} \pi \mathfrak{b})$ then $V(\mathfrak{a})=V(\mathfrak{b})$, while if $\mathfrak{a}$ and $\mathfrak{b}$ are irreducible normal ideals such that $V(\mathfrak{a})=V(\mathfrak{b})$ then it is shown that $\mathfrak{a} \pi \mathfrak{b}$.

Presented to the Society, September 2, 1960 under the title Normal ideals in local domains; received by the editors June 6, 1961. 
2. The proof of the existence of derived normal ideals is a paraphrase of Zariski's proof of the existence of normal varieties. First let $\mathrm{o}$ be a complete local domain integrally closed in its quotient field $F$ and assume that $\mathrm{D}$ contains an infinite field $k$. Let $\mathfrak{a}=\left(a_{1}, a_{2}, \cdots, a_{n}\right)$ be an $D$-ideal and consider the restricted Rees ring

$$
R=\mathrm{o}\left[t a_{1}, t a_{2}, \cdots, t a_{n}\right],
$$

where $t$ is an indeterminate over $F$. An element $\theta$ of $F(t)$ that is integral over $R$ must belong to $\mathrm{o}[t]$ and if $\theta=\sum \theta_{i} t^{i}$, the components $\theta_{i} t^{i}$ of $\theta$ are integral over $R$ since $k$ is infinite. In particular, the element $\theta_{i}$ belongs to the integral closure $\left(\mathfrak{a}^{i}\right)_{a}$ of $\mathfrak{a}^{i}$. By a result of Rees [4], the fact that 0 is analytically unramified implies that there is an integer $h$ such that $\left(a^{n}\right)_{a} \subseteq a^{n-h}$ for all $n \geqq h$, so that if $i \geqq h$, we have $\theta_{i} t^{i} \in R t^{h}$. Hence if $\bar{R}$ is the integral closure of $R$ in $F(t), \bar{R} \subseteq R$ $+R t+\cdots+R t^{h}$ so that $\bar{R}$ is a finite $R$-module. ${ }^{1}$ Moreover, since each integral element is the sum of homogeneous integral elements, we have $\bar{R}=\mathrm{o}\left[z_{1} t^{e_{1}}, \cdots, z_{s} t^{t_{s}}\right]$, where $z_{i}=a_{i}, e_{i}=1$ if $i=1,2, \cdots, n$. Hence if $g$ is a character of homogeneity (see [8]) associated with the integers $e_{1}, e_{2}, \cdots, e_{s}$, and if $T=0\left[w_{1} t^{o}, \cdots, w_{N} t^{o}\right]$, is the ring generated over $\mathrm{D}$ by the products of the $z_{i} t^{t_{i}}$ of degree $g$, then $T$ is integrally closed in its quotient field $F\left(t^{0}\right)$, and it follows readily that the ideal $\mathfrak{b}=\sum o w_{i}$ is normal. Since $\mathfrak{a}^{\circ} \subseteq \mathfrak{b} \subseteq\left(\mathfrak{a}^{o}\right)_{a}$ and since $\mathfrak{b}$ is integrally closed, it follows that $\mathfrak{b}=\left(\mathfrak{a}^{o}\right)_{a}$.

It is easily verified that if $\mathrm{D}$ is a normal domain that is analytically normal and $\hat{b}$ is its completion, then for any primary ideal $a$ belonging to the maximal ideal of $\mathfrak{0}$, the operation of integral closure ( $a$-operation) commutes with the operations of extension and contraction between $\boldsymbol{D}$ and $\hat{\boldsymbol{b}}$, so that we have proved

Proposition 1. If $\mathrm{o}$ is a normal local domain that is analytically normal and contains an infinite field, and if $a$ is an 0 -ideal that is primary for the maximal ideal of $\mathrm{D}$, then there exists an integer $\mathrm{g}$ such that $\mathfrak{a}_{o}=\left(\mathfrak{a}^{0}\right)_{a}$ is a normal ideal.

3. Assume that $\mathrm{D}$ is a normal domain with an infinite residue field $k$ and fix a basis $\alpha_{1}, \alpha_{2}, \cdots, \alpha_{n}$ for the $m$-primary ideal $a$. Let $R$ and $\bar{R}$ denote the polynomial rings $\mathrm{o}\left[X_{1}, \cdots, X_{n}\right]$ and $k\left[X_{1}, \cdots, X_{n}\right]$ respectively, where $X_{1}, X_{2}, \cdots, X_{n}$ are indeterminates. If $\phi \in R$, let $\bar{\phi}$ be the element of $\bar{R}$ obtained from $\phi$ by reading its coefficients modulo $\mathfrak{m}$. A form $\Phi_{s}(X) \in \bar{R}$ of degree $s$ is called a null-form for $a$ if it is the image of a form $\phi_{s}(X)$ in $R$ such that $\phi_{s}(\alpha) \in a^{s} \cdot \mathfrak{m},[3]$. The

1 The use of the Rees theorem here was suggested to us by M. Sakuma. It has resulted in a simplification of our original proof. 
set of all null-forms for $\mathfrak{a}$ generates a homogeneous ideal $\mathfrak{n}(\mathfrak{a})$, and the variety of this ideal in the projective space $P_{n-1}(k)$ will be denoted by $N(\mathfrak{a})$.

Let $\left(c_{1}, c_{2}, \cdots, c_{n}\right)$ be an algebraic point of $N(\mathfrak{a})$ so that when defined, the ratios $c_{i} / c_{j}$ are elements of $\bar{k}$, the algebraic closure of $k$. Assume for example that $c_{1} \neq 0$ and let $\bar{G}_{2}\left(Y_{2}\right), \bar{G}_{3}\left(Y_{2}, Y_{3}\right), \cdots$, $\bar{G}_{n}\left(Y_{2}, Y_{3}, \cdots, Y_{n}\right)$ be Zariski's canonical basis for the nonhomogeneous ideal of the point $\left(c_{2} / c_{1}, \cdots, c_{n} / c_{1}\right)$, [9]. If $\nu$ is the maximum degree of the polynomials $\bar{G}_{i}$, let $\bar{g}_{i}$ be the form of degree $\nu$ obtained from $\bar{G}_{i}$ by replacing each $Y_{j}$ by $X_{j} / X_{1}$ and multiplying by $X_{1}^{p}$. Finally, let $g_{i}$ be a form of degree $\nu$ in $R$ that reduces to $\bar{g}_{i}$ when taken modulo $\mathfrak{m}$, and denote the ideal $\mathfrak{m a}^{p}+o g_{2}(\alpha)+\cdots+o g_{n}(\alpha)$ by $\mathrm{t}$. If $\theta \in \mathfrak{a}^{\nu 0}$, there is a form $\phi(X)$ in $R$ of degree $\nu g$ such that $\theta-\phi(\alpha) \in m a^{\prime \sigma}$. The representative $\phi(X)$ of such a form is unique modulo $\mathfrak{n}(\mathfrak{a})$ and we denote it by $\theta_{0}(X)$.

Lemma 3.1. If $\theta \in \mathfrak{a}^{\nu 0}$, then there is an integer $\mu$ such that $\alpha_{1}^{\nu \mu} \theta$ $\in \mathrm{ta}^{\nu(0-1+\mu)}$, if and only if $\theta_{0}(X)$ vanishes at $\left(c_{1}, c_{2}, \cdots, c_{n}\right)$.

Proof. Assume that $\theta_{0}(c)=0$. The polynomial $\theta_{0}\left(1, Y_{2}, \cdots, Y_{n}\right)$ vanishes at $\left(c_{2} / c_{1}, \cdots, c_{n} / c_{1}\right)$ so that there exist polynomials $f_{2}(Y)$, $\cdots, f_{n}(Y)$ such that $\theta_{0}(1, Y)=\Sigma f_{i}(Y) \bar{G}_{i}(Y)$. Let $q$ be the least integer such that $(q+g-1) \nu$ is not less than the degree of any $f_{i}$. Then we find $X_{1}^{\alpha v} \theta_{0}(X)=\Sigma f_{i}^{*}(X) \bar{g}_{i}(X)$, where $f_{i}^{*}$ is the form of degree $(q+g-1) \nu$ obtained from $f_{i}$. Hence $\alpha_{1}^{q \nu} \phi(\alpha) \in \mathfrak{a}^{(q+o-1)} v$, and $\alpha_{1}^{q \nu} \theta$ $\in \mathfrak{a}^{(0-1+q)} \mathrm{t}$. Conversely, assume that $\alpha_{1}^{\nu q} \theta \in \mathfrak{a}^{(0-1+q)} \mathfrak{t}$. Then there exist forms $F_{2}(X), \cdots, F_{n}(X)$ of degree $\nu(g-1+q)$ such that $\alpha_{1}^{\nu q} \theta-\Sigma F_{i}(\alpha) g_{i}(\alpha) \in m a^{\nu(\theta-1+q)}$. It follows that $X_{1}^{v q} \theta_{0}(X)-\Sigma \bar{F}_{i}(X) \bar{g}_{i}(X)$ is a null-form and hence vanishes at $\left(c_{1}, c_{2}, \cdots, c_{n}\right)$. Since $c_{1} \neq 0$, this implies that $\theta_{0}(c)=0$, q.e.d.

It follows from Lemma 3.1 that if $\theta_{1} \in a^{v o}$ and $\theta_{2} \in a^{\text {ph }}$ are such that $\alpha_{1}^{q \nu} \theta_{1} \notin a^{(o+q-1)} \mathfrak{t}$ and $\alpha_{1}^{q \nu} \theta_{2} \notin a^{\nu(h+q-1)} \mathfrak{t}$ for all positive integers $q$, then also $\alpha_{1}^{q \nu} \theta_{1} \theta_{2} \notin a^{(h+o+q-1)} t$ for all positive integers $q$, so that the set $Z=\left\{\alpha / \beta ; \alpha, \beta \in \mathfrak{a}^{\nu \sigma}, \alpha_{1}^{q \nu} \beta \in \mathfrak{a}^{\nu(o+q-1)} \mathrm{t}, \forall q\right\}$ is a subring of the quotient field $F$ of $\mathrm{o}$. Moreover, the ring $Z$ contains the ring

$$
\mathfrak{D}^{*}=\mathfrak{o}\left[\omega_{1} / \alpha_{1}^{\prime}, \cdots, \omega_{N} / \alpha_{1}^{\prime}\right],
$$

where $\omega_{1}, \omega_{2}, \cdots, \omega_{N}$ is the set of monomials of degree $\nu$ in $\alpha_{1}, \alpha_{2}$, $\cdots, \alpha_{n}$. (Hence in particular, if $\alpha_{1}$ is superficial relative to $a$ then $D^{*}$ is the ring $D\left(a, \alpha_{1}^{\nu}\right)$.)

LeмmA 3.2. If $u_{1}, u_{2}, \cdots, u_{s}$ is a basis for $\mathfrak{m}$, then the ideal generated in $0^{*}$, by $u_{1}, u_{2}, \cdots, u_{s}$ and $g_{1}(\alpha) / \alpha_{1}^{\nu}, \cdots, g_{n}(\alpha) / \alpha_{1}^{y}$ is a maximal ideal $\mathfrak{m}^{*}$ of $\mathrm{o}^{*}$ such that $\mathrm{m}^{*} \cap_{\mathrm{D}}=\mathrm{m}$ and $Z$ is the quotient ring of $\mathrm{o}^{*}$ at $\mathrm{m}^{*}$. 
Proof. The ideal $\mathrm{m}^{*}$ cannot be the unit ideal in $\mathrm{o}^{*}$ for this would imply that for some integer $q, \alpha_{1}^{q \nu} \in \mathfrak{a}^{(q-1)} \mathfrak{t}$, in contradiction with Lemma 3.1. Similarly, the ideal $\mathrm{m}^{*} Z$ is not the unit ideal in $Z$. Moreover, an element $\alpha / \beta$ of $Z$ is a nonunit of $Z$ if and only if $\alpha_{1}^{\nu q} \alpha$ $\in a^{p(q+o-1)} t$ and hence $\alpha / \beta \in \mathfrak{m}^{*} Z$. Thus $Z$ is a local ring and is clearly the quotient ring of $\mathfrak{o}^{*}$ at $\mathrm{m}^{*}$. If $\mathrm{o}_{1}=\mathfrak{D}\left[\alpha_{2} / \alpha_{1}, \cdots, \alpha_{n} / \alpha_{1}\right]$ then $\mathrm{D}^{*}=\mathrm{D}_{1}$ and it is clear that $\mathrm{D}_{1} / \mathrm{m}^{*} \cong k\left(c_{2} / c_{1}, \cdots, c_{n} / c_{1}\right)$ so that $\mathrm{m}^{*}$ is maximal, q.e.d.

Since $k$ is infinite we can select a minimal base $\beta_{1}, \beta_{2}, \cdots, \beta_{r}$ of a minimal reduction of $\mathfrak{a}$ such that each $\beta_{i}$ is superficial relative to $\mathfrak{a}$ (see [3 and 6]). It is assumed that the first $r$ elements $\alpha_{1}, \cdots, \alpha_{r}$ of the $a$-basis above have these properties. Then at each algebraic point $C=\left(c_{1}, \cdots, c_{r}\right)$ of $N(\mathfrak{a})$ at least one of the first $r$ coordinates $c_{i}$ is different from zero. In fact, if $c_{1}=c_{2}=\cdots=c_{r}=0$, then it is clear that $v\left(\alpha_{i}\right)>v(\mathfrak{a})(i=1,2, \cdots, r)$ in any valuation $v$ that dominates the local ring $Z$ associated with $C$ and this is not possible if $\alpha_{1}, \alpha_{2}, \cdots, \alpha_{r}$ generate a reduction of $a$, [3]. Hence it follows from Lemma 3.2 that the ring $Z$ is a quotient ring of one of the rings $\mathfrak{o}\left(\mathfrak{a}, \alpha_{1}\right), \mathfrak{b}\left(\mathfrak{a}, \alpha_{2}\right), \cdots, \mathfrak{o}\left(\mathfrak{a}, \alpha_{r}\right)$ at a maximal ideal $\mathfrak{m}^{*}$ such that $\mathfrak{m}^{*} \cap_{\mathfrak{D}}=\mathfrak{m}$. Thus $Z$ is an $\mathfrak{a}$-spot of maximal rank and the function $f$ defined by $f(C)=Z$ maps the algebraic points of $N(\mathfrak{a})$ into the set of $a$-spots of maximal rank. Moreover, it is clear that if $f(C)=f\left(C^{\prime}\right)$ then $C$ and $C^{\prime}$ have the same ideal in $k\left[X_{1}, \cdots, X_{n}\right]$, so that they are $k$-isomorphic points of $N(\mathfrak{a})$. Hence if $k$-isomorphic points are identified then $f$ is injective.

Lemma 3.3. If an a-spot $P$ contains one of the rings $\mathfrak{D}\left(\mathfrak{a}, \alpha_{i}\right)$, $i=1,2, \cdots, r$, say $\mathfrak{o}\left(\mathfrak{a}, \alpha_{1}\right)$, then $P$ is a quotient ring of $\mathfrak{o}\left(\mathfrak{a}, \alpha_{1}\right)$. If $v \in \Omega$ is such that its valuation ring dominates $P$ and if $v\left(\alpha_{i}\right)=v(\mathfrak{a})$, then $\mathrm{o}\left(\mathfrak{a}, \alpha_{i}\right) \subseteq P$.

Proof. By definition $P$ is a quotient ring of a ring $\mathfrak{D}\left(\mathfrak{a}^{p}, \alpha\right)$ and hence $\alpha_{1}^{\nu} / \alpha \in P$. However, $\mathfrak{o}\left(\mathfrak{a}, \alpha_{1}\right) \subseteq P$ so that $\alpha / \alpha_{1}^{\nu} \in P, \alpha / \alpha_{1}^{\nu}$ is a unit of $P$ and the first assertion of the lemma follows immediately. If say $v\left(\alpha_{1}\right)=v(\mathfrak{a})$ then $v\left(\alpha_{1}^{\nu}\right)=v\left(\mathfrak{a}^{\nu}\right)=v(\alpha)$ and hence $\alpha_{1}^{\nu} / \alpha$ is a unit in $P$. Hence $\alpha_{j} / \alpha_{1} \in P$, and thus $\mathfrak{o}\left(\mathfrak{a}, \alpha_{1}\right) \supseteq P$.

Corollary. Any a-spot $P$ is a quotient ring of one of the rings $\mathrm{o}\left(\mathfrak{a}, \alpha_{1}\right), \mathrm{o}\left(\mathfrak{a}, \alpha_{2}\right), \cdots, \mathfrak{o}\left(\mathfrak{a}, \alpha_{r}\right)$.

If $P$ is an $\mathfrak{a}$-spot of maximal rank we can assume $P$ to be a quotient ring of say $\mathfrak{o}\left(\mathfrak{a}, \alpha_{1}\right)$ at a maximal ideal $\mathfrak{m}^{*}$ that lies over $\mathfrak{m}$. The $\mathfrak{m}^{*}$-residue $c_{j}$ of the quotient $\alpha_{j} / \alpha_{1}$ is then algebraic over $k$ $(j=2,3, \cdots, n)$ and the point $C=\left(1, c_{2}, \cdots, c_{n}\right)$ belongs to the 
variety $N(\mathfrak{a})$. If $f(C)=Z$, then $Z$ is an $\mathfrak{a}$-spot that is a quotient ring of $\mathfrak{o}\left(\mathfrak{a}, \alpha_{1}\right)$ so that clearly $Z=P$. We have thus proved

Proposition 2. There is a 1:1 correspondence between the classes of $k$-equivalent algebraic points of the variety $N(\mathfrak{a})$ and the set of a-spots of maximal rank.

We conclude this section with the remark that the variety $V(\mathfrak{a})$ is necessarily complete with respect to the set $\Omega$ of valuations. Indeed, if $v \in \Omega$ then for some $i \leqq r, v\left(\alpha_{i}\right)=v(\mathfrak{a})$ and $\mathfrak{o}\left(\mathfrak{a}, \alpha_{i}\right)$ is contained in the valuation ring $R_{v}$ of $v$. If $\mathfrak{p}$ is the center of $v$ in $\mathfrak{D}\left(\mathfrak{a}, \alpha_{i}\right)$ then $v$ dominates the $\mathfrak{a}$-spot $\mathfrak{o}\left(\mathfrak{a}, \alpha_{i}\right)_{\mathfrak{p}}$.

4. If $\mathfrak{a}$ and $\mathfrak{b}$ are $\mathfrak{m}$-primary ideals of the local domain $(\mathfrak{b}, \mathfrak{m})$ we say that $\mathfrak{a}$ dominates $\mathfrak{b}$ (in symbols: $\mathfrak{a} \geqq \mathfrak{b}$ ) if each $\mathfrak{a}$-spot dominates some $\mathfrak{b}$-spot or equivalently if the variety $V(\mathfrak{a})$ dominates $V(\mathfrak{b})$. If $\mathfrak{a} \geqq \mathfrak{b}$ and $P$ is a $\mathfrak{b}$-spot, then there is an $\mathfrak{a}$-spot that dominates $P$. In fact, if $v$ is an element of $\Omega$ that dominates $P$, then there is an $\mathfrak{a}$-spot $Q$ such that $v$ dominates $Q$. Since $Q$ dominates a b-spot $P^{\prime}, v$ must dominate $P$ and $P^{\prime}$ so that by Lemma 3.3 these spots are quotient rings of one and the same ring $\mathfrak{D}\left(\mathfrak{a}, \alpha_{i}\right)$. It follows that $P=P^{\prime}$ since they must both be quotient rings at the center $\mathfrak{p}$ of $v$ in $\mathfrak{D}\left(\mathfrak{a}, \alpha_{i}\right)$. This argument shows, incidentally, that the $a$-spot dominated by a given element $v$ of $\Omega$ is unique. ${ }^{2}$

If $(0, \mathfrak{m})$ satisfies the hypothesis of Proposition 1 , then it is easy to see that if $\mathfrak{a}_{o}$ is a derived normal ideal of $\mathfrak{a}$ then $\mathfrak{a}_{o} \geqq \mathfrak{a}$. For this purpose we need a lemma similar to [2, Proposition 1].

LEMMA 4.1. Let a be normal and let $\alpha_{1}, \alpha_{2}, \cdots, \alpha_{r}$ be a minimal base of a minimal reduction of $a$. If $\pi$ is the prime field of $\mathrm{o}$ and $\phi(X)$ is a nonzero form of degree $t$ in $\pi\left[X_{1}, X_{2}, \cdots, X_{r}\right]$, then $\mathfrak{a}^{n}: \mathbb{D} \phi(\alpha)=\mathfrak{a}^{n-t}$ for all $n \geqq t$.

Proof. Pass to the completion $\hat{\mathfrak{b}}$ of $\mathfrak{o}$, let $k$ be a coefficient field of $\hat{\mathbf{v}}$ and form the power series ring $Q=k\left\{\alpha_{1}, \alpha_{2}, \cdots, \alpha_{r}\right\}$. Then $\hat{\mathbf{v}}$ is a finite $Q$-module and it is easy to see that the integral dependence of an element $\omega$ upon $\hat{\mathfrak{a}}^{n}(\hat{\mathfrak{a}}=\hat{\mathbf{b}} \mathfrak{a})$ is put into evidence by the minimal equation of $\omega$ over $Q$ (see [2, Lemma 1]). Hence if $\phi \in \pi[X]$ and $\phi(\alpha) \omega \in \mathfrak{a}^{n}$ then $\omega$ must depend integrally upon $\mathfrak{a}^{n-t}$. In fact, if $a_{i}$ is the coefficient of $\omega^{\delta-i}$ in the minimal polynomial of $\omega$ over $Q$, then $\phi(\alpha)^{i} a_{i}$ is the corresponding coefficient in the minimal polynomial of $\phi(\alpha) \omega$, and if $A=\mathfrak{a} \cap Q$, the fact that $\phi^{i} a_{i} \in A^{n i}$ implies that $a_{i} \in A^{n i-t i}$, since $Q$ is regular. Since $\mathfrak{a}$ is normal, $\omega \in \mathfrak{a}^{n-t}$, q.e.d.

2 The referee has kindly pointed out to us that in view of this fact and the Corollary to Lemma 3.3 , the local variety $V(\mathfrak{a})$ is a complete (proper) schema in the sense of Grothendieck. See [1, Ch. II, \$7]. 
Now let $\alpha_{1}, \cdots, \alpha_{r}$ be a minimal base of a minimal reduction of $a$ and let $\beta_{i}=\alpha_{i}^{q}$. Then $\beta_{1}, \cdots, \beta_{r}$ is a minimal base of a minimal reduction of $\mathfrak{a}^{o}$ and hence also of $\mathfrak{a}_{0}$. By Lemma 4.1 each $\beta_{i}$ is superficial relative to $\mathfrak{a}_{g}$. Moreover, $\mathfrak{D}\left(\mathfrak{a}_{a}, \beta_{i}\right)$ is the integral closure of $\mathfrak{o}\left(\mathfrak{a}^{o}, \beta_{i}\right)$ in its quotient field. Hence each spot of $\mathfrak{a}_{\boldsymbol{v}}$ dominates a spot of $\mathfrak{a}$ and since $\mathfrak{o}\left(\mathfrak{a}_{g}, \beta_{i}\right)$ is a finite module over $\mathfrak{D}\left(\mathfrak{a}^{\mathfrak{o}}, \beta_{i}\right)$ the integral closure of an $a$-spot $P$ is the intersection of a finite number of $\mathfrak{a}_{0}$-spots that dominate $P$. Thus $V\left(\mathfrak{a}_{g}\right)$ is a normalization of $V(\mathfrak{a})$.

As mentioned above, an $a$-spot $P$ is a specialization of an $a$-spot $Q$ if $Q$ is a quotient ring of $P$. If $\mathfrak{p}_{i 1}, \mathfrak{p}_{i 2}, \cdots, \mathfrak{p}_{i s_{i}}$ is the set of minimal prime ideals of the principal ideal $\alpha_{i} \mathrm{o}\left(\mathfrak{a}, \alpha_{i}\right)$, and if $P_{i 1}, P_{i 2}$, $\cdots, P_{i s_{i}}$ are the corresponding a-spots, then each a-spot $P$ is a specialization of at least one of the spots $P_{i j}$. In fact, if $\mathfrak{p}$ is a prime ideal in $\mathfrak{o}\left(\mathfrak{a}, \alpha_{i}\right)$ such that $\mathfrak{p} \cap \mathbb{D}=\mathfrak{m}$ then $\alpha_{i} \in \mathfrak{p}$ and hence $\mathfrak{p} \supseteq \mathfrak{p}_{i j}$ for some $j$. If $\mathfrak{a}$ is a normal ideal $\mathfrak{D}\left(\mathfrak{a}, \alpha_{i}\right)$ is integrally closed and each $P_{i j}$ is the valuation ring of a discrete archimedean valuation of the quotient field $F$ of $\mathfrak{D}$. These are precisely the valuation rings of the valuations that occur in the Rees representation of the homogeneous pseudo-valuation $\bar{v}_{\mathfrak{a}}$ associated with $\mathfrak{a}[5]$. It is natural to say that an $\mathfrak{a}$-spot $P$ is generic for $\mathfrak{a}$ if all $\mathfrak{a}$-spots are specializations of $P$ and to call an $\mathfrak{m}$-primary ideal $\mathfrak{a}$ irreducible if it admits a generic spot. An ideal that is irreducible in our sense need not be irreducible as an element of the ideal semi-group under the intersection composition. We will show in a later paper, however, that an irreducible ideal as defined here is either simple in the sense of Zariski [10, Appendix 5] or is a power of a simple ideal.

Proposition 3. Let $(\mathfrak{o}, \mathrm{m})$ be a local domain which satisfies the conditions of Proposition 1, let $\mathfrak{a}$ and $\mathfrak{b}$ be normal $\mathfrak{m}$-primary ideals of $\mathfrak{o}$, and let $\mathrm{c}$ be a derived normal ideal of the product $\mathfrak{a} \cdot \mathfrak{b}$. Then $\mathfrak{c} \geqq \mathfrak{a}, \mathfrak{c} \geqq \mathfrak{b}$, and if $\mathfrak{n}$ is any normal ideal that dominates both $\mathfrak{a}$ and $\mathfrak{b}$ then $\mathfrak{n} \geqq \mathfrak{c}$.

Proof. Let $v_{1}, v_{2}, v_{3}$ be the homogeneous pseudo-valuations associated with $\mathfrak{a}, \mathfrak{b}$, and $\mathfrak{c}$ in the sense of Rees [5]. Each $v_{i}$ can be represented as a minimum of a finite set of valuations: $v_{i}(x)$ $=\min \left\{v_{i 1}(x) / e_{i 1}, \cdots, v_{i s_{i}}(x) / e_{i s_{i}}\right\}, i=1,2,3$, where $e_{i j}$ is an integer and $v_{i j}$ is an integer valued element of $\Omega$. This representation holds for all $x$ in 0 . Let $W$ be the set of valuations $v_{i j}\left(i=1,2,3 ; 1 \leqq j \leqq s_{i}\right)$. Let $P$ be a c-spot and let $v$ be an element of $\Omega$ such that $R_{v}$ dominates $P$. Select elements $\alpha \in \mathfrak{a}, \beta \in \mathfrak{b}$ such that $v(\alpha)=v(\mathfrak{a}), v(\beta)=v(\mathfrak{b})$ and $v_{i j}(\alpha)=v_{i j}(\mathfrak{a}), v_{i j}(\beta)=v_{i j}(\mathfrak{b})$ for all $v_{i j} \in W$. (Since $\mathrm{o}$ has an infinite residue field the existence of such elements is trivial.) Since $a$ is normal the conditions $v_{i j}(\alpha)=v_{i j}(\mathfrak{a})$ imply that $\alpha$ is superficial relative to a. In fact, $\alpha x \in \mathfrak{a}^{n}$ implies that $v_{i j}(x) \geqq(n-1) v_{i j}(\mathfrak{a})$, and hence 
$v_{1}(x) \geqq n-1$. Since $\mathfrak{a}^{n-1}$ is integrally closed, $x \in \mathfrak{a}^{n-1}$. Similarly, $\beta$ is superficial relative to $\mathfrak{b}$ and if $g$ is such that $c=\left(\mathfrak{a}^{0} \cdot \mathfrak{b}^{0}\right)_{a}$, then $\alpha^{0} \cdot \beta^{o}$ is superficial relative to $c$. Moreover, since $v\left(\alpha^{g} \beta^{o}\right)=v(c)$, the ring $\mathrm{D}\left(\mathrm{c}, \alpha^{a} \beta^{o}\right)$ is contained in $R_{v}$ and $P$ is a quotient ring of $\mathrm{D}\left(\mathrm{c}, \alpha^{a} \beta^{o}\right)$. At the same time each of the rings $\mathfrak{o}(\mathfrak{a}, \alpha)$ and $\mathfrak{D}(\mathfrak{b}, \beta)$ is a subring of $\mathfrak{D}\left(\mathfrak{c}, \alpha^{a} \beta^{o}\right)$, so that $P$ dominates an $\mathfrak{a}$-spot and a $\mathfrak{b}$-spot. Since any integrally closed ring that contains both $\mathfrak{D}(\mathfrak{a}, \alpha)$ and $\mathfrak{o}(\mathfrak{b}, \beta)$ must also contain $D\left(c, \alpha^{a} \beta^{o}\right)$, the second assertion of the proposition is clear.

Proposition 4. Under the hypotheses of Proposition 3, if $\mathfrak{a} \pi \mathfrak{b}$ then $V(\mathfrak{a})=V(\mathfrak{b})$. If $\mathfrak{a}$ and $\mathfrak{b}$ are normal and irreducible, then $V(\mathfrak{a})=V(\mathfrak{b})$ implies $\mathfrak{a} \pi \mathfrak{b}$.

Proof. Let the Samuel functions $l_{\mathfrak{a}}(\mathfrak{b})$ and $L_{\mathfrak{a}}(\mathfrak{b})$ (see [7]) have the common value $p / q$. Then $l_{\mathfrak{a}^{p}}\left(\mathfrak{b}^{q}\right)$ and $L_{\mathfrak{a}^{p}}\left(\mathfrak{b}^{q}\right)$ have the common value 1 and $\mathfrak{a}^{p}$ and $\mathfrak{b}^{\mathfrak{a}}$ are integrally equivalent. Since $\mathfrak{a}$ and $\mathfrak{b}$ are normal, $\mathfrak{a}^{p}=\mathfrak{b}^{\mathfrak{a}}$, and $V(\mathfrak{a})=V(\mathfrak{b})$. Conversely, if $\mathfrak{a}$ and $\mathfrak{b}$ are irreducible the homogeneous pseudo-valuations $\bar{v}_{\mathfrak{a}}$, and $\bar{v}_{\mathfrak{b}}$ are valuations, and the equality $V(\mathfrak{a})=V(\mathfrak{b})$ implies that these valuations have the same valuation ring. Hence there is an integer valued valuation $v$ of the quotient field of $\mathfrak{D}$ such that $\bar{v}_{\mathfrak{a}}(x)=v(x) / e$ and $\bar{\nu}_{\mathfrak{b}}(x)=v(x) / f$ for all $x$ in $\mathfrak{0}$. Here $e$ and $f$ are integers and $e=v(\mathfrak{a}), f=v(\mathfrak{b})$. It follows that $\mathfrak{a}^{e}$ and $\mathfrak{b}^{f}$ determine the same homogeneous pseudo-valuation and are therefore integrally equivalent and hence equal, q.e.d.

\section{BIBLIOGRAPHY}

1. A. Grothendieck, Elements de geometrie algébrique, IHES Pub. Math. No. 8, 1961.

2. H. T. Muhly, Complete ideals in local rings, Proc. Amer. Math. Soc. 11 (1960), 361-367.

3. D. G. Northcott-D. Rees, Reductions of ideals in local rings, Proc. Cambridge Philos. Soc. 50 (1954), 145-158.

4. D. Rees, $A$ note on analytically unramified local rings, J. London Math. Soc. 36 (1961), 24-28.

5. - Valuations associated with ideals, Proc. London Math. Soc. 6 (1956), 161-174.

6. P. Samuel, Algibre locale, Gauthier-Villars, Paris, 1953.

7. - Some asymptotic properties of powers of ideals, Ann. of Math., 56 (1952), $11-21$

8. O. Zariski, Some results in the arithmetic theory of algebraic varieties, Amer. J. Math. 61 (1939), 249-294.

9. - The concept of a simple point of an abstract algebraic variety, Trans. Amer. Math. Soc. 62 (1947), 1-52.

10. O. Zariski, and P. Samuel, Commutative algebra, Vol. 2, D. Van Nostrand, Princeton, 1960.

State University of Jowa 